OPEN ACCESS

Edited by:

Marientina Gotsis, University of Southern California,

United States

Reviewed by:

Sheila A. M. Rauch,

Emory University, United States

Randy Klaassen,

University of Twente, Netherlands

Raul Espert-Tortajada,

University of Valencia, Spain

*Correspondence:

Ray Jackson

info@neuromeditationinstitute.com

Specialty section:

This article was submitted to Virtual Reality in Medicine, a section of the journal Frontiers in Virtual Reality

Received: 25 August 2021 Accepted: 06 January 2022 Published: 26 January 2022

Citation:

Tarrant J, Jackson $R$ and Viczko J (2022) A Feasibility Test of a Brief Mobile Virtual Reality Meditation for Frontline Healthcare Workers in a Hospital Setting.

Front. Virtual Real. 3:764745. doi: 10.3389/frvir.2022.764745

\section{A Feasibility Test of a Brief Mobile Virtual Reality Meditation for Frontline Healthcare Workers in a Hospital Setting}

\author{
Jeff Tarrant ${ }^{1}$, Ray Jackson ${ }^{1 *}$ and Jeremy Viczko ${ }^{2}$ \\ ${ }^{1}$ NeuroMeditation Institute, Eugene, OR, United States, ${ }^{2}$ Department of Psychology, University of Victoria, Victoria, BC, Canada
}

The purpose of this study was to examine whether a virtual reality plus neurofeedback (VR+NF) meditation experience (experimental condition) was more effective than a standard guided audio-only meditation (control condition) in improving mood in one hundred healthcare workers. Data collection occurred in a hospital setting between October, 2020 and March, 2021 at the height of the COVID-19 pandemic. Participants were alternately assigned to one of the two conditions. Before and after the meditation experience, participants completed the Brunel Mood Scale. Results indicated that both groups showed a similar and significant decrease in Anger, Tension, and Depression. On scales measuring Vigor, Fatigue, and Confusion, the VR+NF group showed decreases, while the audio-only group showed no significant change. The VR+NF group showed significant increases on the Calmness and Happiness scales, which did not change significantly in the audio-only group. These results suggest that the addition of VR and neurofeedback may increase the positive outcomes associated with standard audioguided meditation. These increased benefits may be due to the sense of presence intrinsic to VR, the inclusion of nature-based scenes in the VR experience, as well as the increased self-awareness created by the addition of neurofeedback. As the pre and post measures take place within one 50-min session, further studies assessing the longer-term changes are needed.

Keywords: virtual reality, meditation, neurofeedback, mental health, apps and smartphones, Mindfulness, Body Scan

\section{INTRODUCTION}

COVID-19, first identified in December of 2019, became a worldwide pandemic causing major disruption and loss of life. The disease is characterized by pneumonia-like symptoms with a high infection and mortality rate. At the time this study was initiated there was no consistent governmental approach to managing the disease in the United States and a vaccine was many months from becoming available. As a result, the presence of this disease caused a dramatic increase in stress, anxiety and psychological concerns (Asmundson and Taylor, 2020; Liu et al., 2020; Taylor et al., 2021). Common fears related to COVID-19 include: becoming infected, coming into contact with possibly contaminated objects, foreigners who might be carrying infection (i.e., disease-related xenophobia), and socio-economic consequences of the pandemic (e.g., job loss; Taylor et al., 2021). Frontline medical staff have been faced with a greater exposure to infection as well as additional 
stressors related to chronic hypervigilance, increased workload, and inconsistent or insufficient access to protective equipment. (Alharbi et al., 2020; Bao et al., 2020; Krystal and McNeil, 2020). Consequently, medical personnel are at significant risk of stressrelated psychological symptoms that may lead to burnout, depression, and post-traumatic stress (Cullen et al., 2020).

A recent survey of medical staff involved in COVID-19 prevention and management indicated extremely high rates of mental health concerns. Half of those surveyed acknowledged symptoms of depression (50.7\%), while 44.7, 36.1, and $73.4 \%$ indicated significant concerns with anxiety, insomnia, and stress symptoms, respectively (Liu et al., 2020). A survey of medical staff in the Wuhan Province during the Covid-19 outbreak showed significant increases in both anxiety and panic related to the dramatic increase of stress (Wang et al., 2020). Similarly, during the SARS outbreak, mental health problems of outpatient medical staff in military hospitals increased dramatically and included somatic complaints, depression, anxiety, and compulsive behaviors (Liu et al., 2004). All of this is consistent with previous studies which have shown that depression and anxiety are common mental health experiences after a disaster (Wang et al., 2000; Fergusson et al., 2014). In response to this potential mental health crisis, there have been calls for increased support and services for healthcare workers (Nica, 2017; Liu et al., 2020). Given the number of people affected, the potential health impacts, and the critical role healthcare workers play in society, it seems important to identify and advance stress-management approaches that can be implemented within a clinical healthcare environment (Richardson and Rothstein, 2008; Chirico et al., 2021). To be successful, such a program would need to be engaging, efficient, convenient, and effective. Based on these criteria, stress management interventions provided through a portable, self-contained virtual reality (VR) device may be an ideal match for this need.

\section{Virtual Reality as a Mental Health Intervention}

VR based interventions have become increasingly popular for mental health and wellness applications (Malbos et al., 2013; Maples-Keller et al., 2017). There have been several reviews examining the potential impact of VR-based treatment approaches for a variety of psychiatric conditions including schizophrenia, substance abuse, eating disorders, and Post Traumatic Stress Disorder (PTSD) (Eichenberg and Wolters, 2012; Veling et al., 2014; Valmaggia et al., 2016; Freeman, et al., 2017; Rus-Calafell et al., 2018; Carl et al., 2019). However, the primary mental health concerns targeted with interventions relate to anxiety-based disorders including phobias and generalized anxiety disorder (GAD; Motraghi et al., 2014; Morina et al., 2015).

For example, a recent meta-analysis of 30 research studies examined the efficacy of VR therapies for a variety of specific phobias. The results indicated that in virtuo treatment with VR resulted in positive outcomes for most of the phobias examined (Frietas et al., 2021). Gorini et al. (2010) and Tarrant et al. (2018) both reported significant reductions in anxiety in those with GAD following nature-based VR experiences. Effective as a coping and therapeutic resource for clinically diagnosed individuals, VR interventions might also be effectively deployed to manage chronic stress and prevent burnout in non-patient populations-such as front-line healthcare workers.

Specifically, VR-based stress management approaches that incorporate meditative practices may be of particular benefit as the impacts of non-VR meditations have been well researched and found to be effective for improving mental health and wellbeing (Goyal et al., 2014) In a review of VR for the treatment of anxiety, Maples-Keller et al. (2017), noted that VR applications that incorporate mindfulness elements may be a particularly useful approach in the treatment of GAD.

\section{Virtual Reality Meditation Made Mobile}

The term meditation is used to refer to a wide range of mental training practices. Researchers have attempted to classify meditation types based on how attention is engaged and which brain regions are active or inhibited during practice (Travis and Shear, 2010; Fox et al., 2016). Mindfulness is often defined as, "the awareness that emerges through paying attention on purpose, in the present moment, and non-judgmentally to the unfolding of experience moment by moment" (Kabat-Zinn, 2003, p. 145). Mindfulness practices are often taught by guiding the meditator toward an awareness of the body or somatic sensations (Williams et al., 2006). This strategy is a key feature of the Mindfulness Based Stress Reduction (MBSR) program and has been studied as part of several mindfulness based programs (Teasdale et al., 2000; Philippot et al., 2012; Evans et al., 2018).

There is a growing body of evidence supporting that mindfulness meditation and other meditation-based programs and services, delivered through mobile devices (e.g., smartphones, tablets etc) are effective in reducing stress, and improving wellbeing (Coulon et al., 2016; Yang et al., 2018). Flett et al. (2019) compared the effectiveness of three meditation-based phone apps in a college student population. All of the apps led to a significant decrease of depressive symptoms and improvements in college adjustment. One app led to improvements in resilience, and another app led to improvements in mindfulness. Carissoli et al. (2015) compared an app-based mindful meditation to a relaxation music control group. Both groups experienced a significant reduction in stress. In two recent studies, Tarrant et al., demonstrated that a brief meditation-based VR experience could be successfully used to reduce state anxiety and increase positive mood in participants with moderate to high generalized anxiety (2018) and just prior to donating blood (2019). These and other studies (e.g., Athanas et al., 2019) suggest the feasibility and promise of meditation training apps for supporting mental health and reducing stress. Whether mobile app-based meditation training is feasible, desired, or effective for supporting the mental health needs of frontline healthcare workers has yet to be determined.

The conventional method of finding a quiet space to listen to a pre-recorded guided meditation may not be possible in a busy, high-demand, high-stress environment. It has been suggested that VR may be particularly suited for creating a meditation environment in situations like hospital settings, that might not 
otherwise be conducive to meditation (Viczko et al., 2021). The sense of presence intrinsic to the VR experience may aid the process of meditation by limiting the amount of information requiring attention and removing external distractions (Slater and Wilbur 1997; Tussyadiah et al., 2018). Beyond the significant perk of being able to quickly and easily "step-out" of one environment, and "step into" a virtual space, it has been suggested that VR has the potential to facilitate aspects of the meditation process itself. For example, specific visual cues provided in the VR context may assist the user in directing attention more successfully than audio-only meditations (Döllinger et al., 2021). Consequently, this approach seems ideal for health care settings.

The VR meditation experience identified for this study also incorporated electroencephalography (EEG) biofeedback. EEG biofeedback or neurofeedback, is a form of brain wave training that provides the user with information about their brainwave activity in near real time (Smith et al., 2014). This feedback, in the form of visual or auditory cues, changes in response to shifts in brainwave activity, increasing awareness of brain behavior and related changes in mental states. With this process, it is possible to guide or encourage the user into an experience of meditation or relaxation (Tarrant 2017).

A study by Garrison et al. (2013) provided visual feedback to meditators about the activity level of the Posterior Cingulate Cortex (PCC) during a focused attention style of meditation. They found that experienced meditators were able to intentionally decrease activation of the PCC through the use of the feedback by engaging in meditation. Van Lutterveld et al. (2017) found that both novice and experienced meditators were able to control the experience of effortless awareness in connection with a feedback signal indicating decreased PCC activation. In another study, participants were asked to engage in a breath-focus meditation practice while either receiving feedback about their frontal midline (FM) Theta activity or sham feedback. Not surprisingly, the group that received accurate feedback learned to intentionally modulate their FM Theta activity while the sham feedback group did not (Brandmeyer and Delorme, 2020). These studies provide evidence that neurofeedback signals can be effectively used to influence a meditator's experience.

The addition of neurofeedback to meditation may provide additional support to meditators by enhancing awareness of inner states (Brandmeyer and Delorme, 2020). In addition, both meditation and neurofeedback have been found to be effective in the treatment of a variety of mental health concerns (Brandmeyer, and Delorme, 2013). Consequently, combining the two may provide a powerful treatment intervention for Attention Deficit Hyperactivity Disorder (ADHD), anxiety, depression, and PTSD (Tarrant, 2017).

Specific to this study, the Healium VR software integrates with a consumer EEG headband to measure brainwave activity in frontal electrodes (FP1, FP2). This information is displayed in the VR screen to provide the user with direct information on changes in the amount of fast brainwave activity (high beta, 18-29.75 Hz) observed moment to moment. Monitoring this particular range of high beta frequencies may be particularly relevant for the current study as increased high beta activity has consistently been identified as a correlate of stress and anxiety (Hammond, 2005; Thompson and Thompson, 2007; Price and Budzynski, 2009; Sherlin et al., 2009; Olbrich et al., 2011). In a study with anxious participants, Tarrant, et al. (2018) found a significant correlation between elevated anxiety and high beta activity. In addition, they found that a VR meditation experience resulted in a significant increase in the low beta/high beta ratio which was not observed in the control condition. Based on this, lower levels of high beta brainwaves could be assumed to reflect decreased levels of stress and anxiety or states of relaxation and meditation (Sherlin et al., 2010).

While the combination of VR with neurofeedback is a relatively new approach, there is preliminary evidence to support its viability and utility. For example, Berger and Davelaar, (2017) demonstrated that frontal alpha EEG signals could be successfully controlled in a VR environment. In addition, they found that changes in the frontal alpha signal were consistent with changes in attentional control and that VR based approaches led to faster learning rates compared to $2 \mathrm{D}$ neurofeedback. A proof of concept study combining VR with neurofeedback demonstrated that the EEG data collected from a consumer headband could be successfully used to assist users in shifting their feeling state (Tarrant and Cope, 2018). Another study, comparing augmented reality with and without neurofeedback, demonstrated that the group receiving neurofeedback found the experience to be more engaging and motivating (Viczko et al., 2021). In a recent case report, Orakpo et al. (2021) demonstrated that VR plus neurofeedback was effective in managing pain, anxiety, depression, and improving sleep in a patient with multiple medical and psychological concerns following a motor vehicle accident. These results suggest that the addition of neurofeedback to VR may enhance aspects of the experience and/or increase user engagement.

\section{Objective}

This study sought to compare the effects of frontline healthcare workers practicing an audio-only guided meditation to a $\mathrm{VR}+$ neurofeedback (VR+NF) guided meditation, made available to them in the hospital workplace setting. The audio only meditation was selected for the control condition as this is the most common platform for guided meditations (Roquet and Corina, 2018). It was hypothesized that both groups would show reductions in negative mood states and increases in positive mood states, but that these changes would be significantly greater in the $\mathrm{VR}+\mathrm{NF}$ group.

This study utilized an alternating assigned between-group experimental design. The two experimental groups were a $\mathrm{VR}+\mathrm{NF}$ delivered meditation and an audio-only meditation, the latter serving as an active control condition.

\section{MATERIALS AND METHODS}

\section{Recruitment}

This study was performed at the Boone Hospital Center in Columbia, MO. It was approved by the Washington 
University Institutional Review Board (St. Louis, MO). Participants were health care workers who were recruited via a hospital newsletter and flyers posted on medical and surgical floors. Participants signed up via the posted email address. Participants were able to choose the day and timing of their participation to suit their schedule while on site for one of their shifts. Bias in participant selection was minimized as the researcher involved in data collection was not involved in scheduling. In addition, screening restrictions, participant cancellations, and schedule changes required that the intervention assignments were not determined until screening and consent were completed.

\section{Inclusion/Exclusion Criteria}

Inclusion criteria required participants to be at least 18 years of age. Exclusion criteria included any history of seizures, current blindness, or severe eye impairment which would limit the ability to engage in the VR experience, or history of motion sickness.

\section{Alternating Assignment}

The participants were alternately assigned to either the VR or audio group based on the order of their consent to participation (i.e. odd numbered participants were in the VR group, while even numbered participants were placed in the audio group.) This resulted in an even sample size, with each condition receiving 50 participants.

\section{Power}

A target sample size of 100 was chosen based on a prior sample size calculation with the software $G^{\star}$ Power 3 (Faul et al., 2007). This analysis determined that a sample size of 84 would have been adequate to detect differences between groups, reaching a statistical power of 0.80 to detect a medium sized effect $(\mathrm{dz}=$ 0.5 ) via a two-tailed dependent means t- test (significance set at $\alpha$ $=05$ ). Thus, the current sample size was equipped to determine both medium and small effect sizes.

\section{Experimental Measures}

\section{Demographic Questionnaire}

This questionnaire asked participants to identify information related to their sex, age, race, education level, experience with meditative practices, and history of mental illness.

\section{Brunel Mood Scale}

The Brunel Mood Scale (Soos et al., 2007) consists of 32 mood descriptors that are categorized into 8 unipolar dimensions: anger, tension, depression, vigor, fatigue, confusion, happiness, and calmness. Each subscale consists of $4 \operatorname{mood}$ descriptors. Using a response frame of "how do you feel right now?" participants indicated the extent of their "in the moment" experience of the mood descriptors on a 5-point scale $(0=$ not at all, $1=$ a little, $2=$ moderately, $3=$ quite a bit, $4=$ extremely). Previous studies of the Brunel Mood Scale have demonstrated a Cronbach Alpha calculation coefficient for internal consistency of 0.87 and test-retest reliability of 0.85 (Çakiroğlu et al., 2020).

\section{Instruments}

\section{Guided Meditation}

The same meditation audio track was used to guide both groups in their meditation. The meditation selected was a progressive body-scan/relaxation mindfulness meditation and is a standard meditation that is offered in the Healium VR "relaxation beach" experience. A woman's voice guided the user through a meditation experience that lasted approximately $5 \mathrm{~min}$, directing their attention to specific parts of the body, inviting relaxation into each part.

\section{Virtual Reality}

The VR experience was "relaxation beach" from the Healium platform (Figure 1) viewed through an Oculus Go VR headset. This experience was $5 \mathrm{~min}, 39 \mathrm{~s}$ in length and produced by Healium XR (Columbia, MO, United States) using $360^{\circ}$ video photography. As the scene opens the user is on a beach with rock outcroppings on either side with waves gently rolling in.

\section{Neurofeedback}

The BrainLink Lite EEG headband (Figure 2) was used to facilitate neurofeedback capabilities in conjunction with the VR environment and guided meditation instructions. The BrainLink Lite headband uses three gold-plated copper sensors situated on the forehead. EEG data is transferred to the Healium platform via Bluetooth, which allows users to control aspects of the VR experience.

This consumer grade headband consists of 2 active electrodes at approximately FP1 and FP2 with the reference at FPZ (on the forehead). Data was sampled at $256 \mathrm{~Hz}$ with the Brainlink Device, then further processed and downsampled by the Healium application in order to provide the user with neurofeedback interfacing. No EEG data was collected or saved during this study. The data was only used for purposes of visual biofeedback and then destroyed within the software.

When this application was interfaced with the Brainlink Lite EEG Headband, the amount of high beta $(18-29.75 \mathrm{~Hz})$ activity was displayed in a simplified form at the bottom of the Healium VR experience. Specifically, the amplitude of high beta activity was represented by a glowing ball that moves up and own in relation to changes in amplitude. This "firefly" moved across the screen in relation to time (See Figure 1). A solid reference or threshold line was displayed in the same space providing a target for the biometric information. This threshold could be adjusted to make the task easier or harder. For the current study, the threshold was adjusted to "easy."

\section{Procedures}

The data was collected in a single 50 -min visit which took place in dedicated research rooms in the hospital. These rooms were on patient floors, but separated from patient rooms to minimize distractions.

\section{Screening}

Upon arrival, participants were given a verbal screening that included the inclusion/exclusion criteria for the study. 


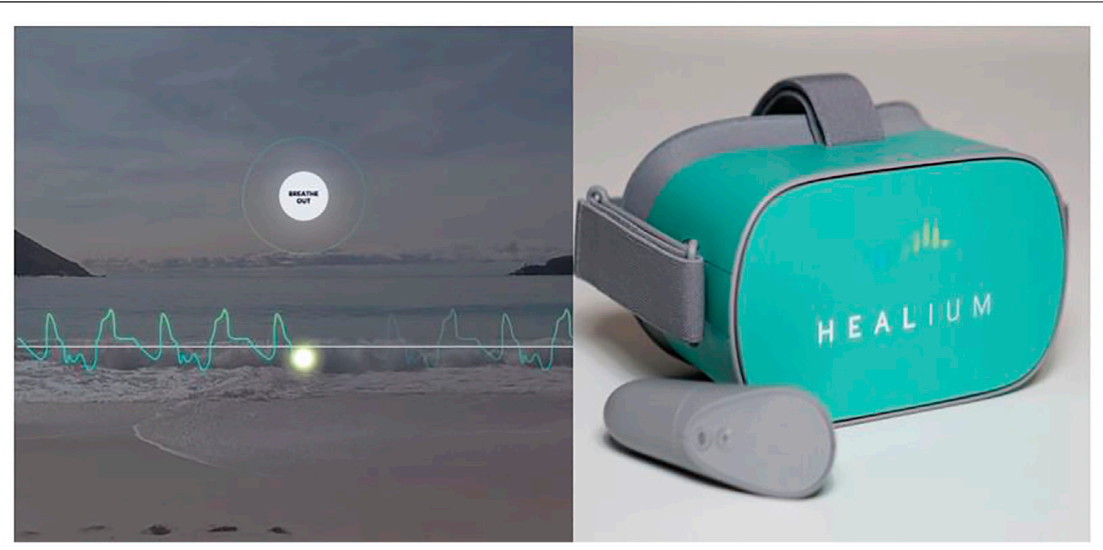

FIGURE 1 | The Healium Oculus Go Virtual Reality Headset and images of the beach scenery. The graph like waves act as neurofeedback for the participant's brainwave patterns.

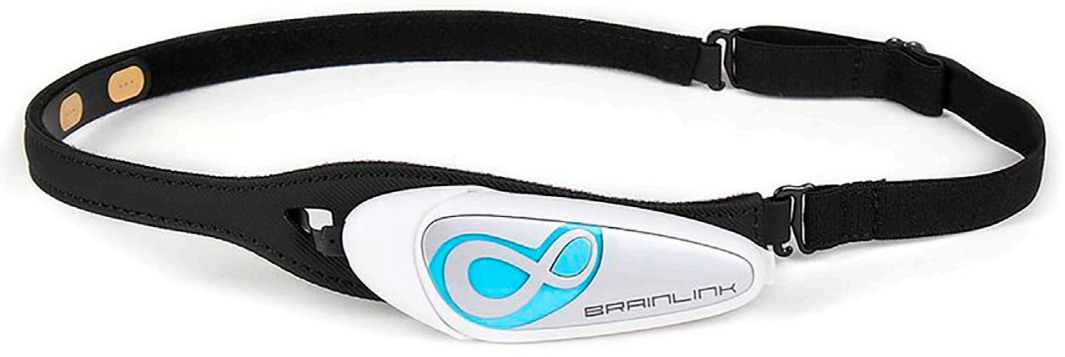

FIGURE 2 | The BrainLink Lite EEG headband, used to facilitate neurofeedback during the experience.

Participants verbally responded "yes" or "no" to the criteria. All participants met the inclusion and exclusion criteria.

\section{Consent and Pre- Intervention Questionnaires}

After completing the verbal screening, participants were provided with an overview of the study that included informed consent.

Participants were told they would be assigned to one of two groups being compared for different meditation experiences. Following informed consent procedures, participants completed via pen and paper a selection of the Brunel Mood Scale subscales measuring "in this moment" mood states. Participants were then directed toward their identified intervention as described below. Immediately following the meditation experience participants repeated the Brunel Mood Scale. During this time, the researcher was present, however, to mitigate researcher influence, their focus was on cleaning equipment, preparing for the next participant, and organization of data.

At the conclusion of the intervention, participants were asked if they experienced any adverse events including discomfort, dizziness, nausea, vomiting, headache and visual disturbance. No adverse events were reported.

\section{Virtual Reality Meditation Condition}

Those in the VR group were then fitted with the Oculus and Brainlink Lite headband. Once fitted, the researcher pressed play on the Oculus VR goggles and allowed the participant to view the experience for 1 minute. At the end of the minute, the researcher asked the participant if they can satisfactorily see the video, and if they are experiencing any nausea or dizziness. If not, the session continued. If the participant expressed any discomfort, the session would be stopped.

Information about the users' brainwave activity was simultaneously provided via a Brainlink Lite EEG headband (see Figure 2) and appeared on the bottom of the VR screen, with their brainwave activity being represented by a glowing firefly. The goal in the current study was to keep the high beta activity below the threshold, indicating a state of inner calm (Sherlin et al., 2010; Tarrant et al., 2018). To make the feedback consistent with descriptions of the activity (increasing calm), the display was reversed, such that success resulted in the firefly moving above the threshold. As long as this criterion was maintained, the VR beach experience continued to play uninterrupted.

When the firefly dropped below the threshold (indicating thinking, analysis, or stress) for 5 consecutive seconds, the screen was tinted with red overlay, indicating to the user that 
TABLE 1 | Participant demographics by group.

\begin{tabular}{|c|c|c|c|c|c|}
\hline & & \multicolumn{2}{|c|}{ Virtual Reality } & \multicolumn{2}{|c|}{ Audio-Only } \\
\hline $\mathrm{N}$ & & \multicolumn{2}{|c|}{50} & \multicolumn{2}{|c|}{50} \\
\hline \multicolumn{6}{|c|}{ Age } \\
\hline & Mean & \multicolumn{2}{|l|}{42.16} & \multicolumn{2}{|l|}{40.9} \\
\hline & Std Deva & \multicolumn{2}{|l|}{14.4} & \multicolumn{2}{|l|}{13.9} \\
\hline \multicolumn{6}{|c|}{ Gender } \\
\hline & Female & 43 & $86 \%$ & 48 & $96 \%$ \\
\hline & Male & 7 & $14 \%$ & 2 & $4 \%$ \\
\hline \multicolumn{6}{|c|}{ Race/Ethnicity } \\
\hline & Caucasian & 49 & $98 \%$ & 48 & $96 \%$ \\
\hline & African & 0 & $0 \%$ & 3 & $6 \%$ \\
\hline & Hispanic & 1 & $2 \%$ & 0 & $0 \%$ \\
\hline & Middle & 0 & $0.0 \%$ & 1 & $2 \%$ \\
\hline & Prefer not to & 0 & $0 \%$ & 0 & $0 \%$ \\
\hline \multicolumn{6}{|c|}{ Education Level } \\
\hline & High School & 1 & $2 \%$ & 0 & $0 \%$ \\
\hline & Some College & 5 & $10 \%$ & 6 & $12 \%$ \\
\hline & Associate's & 6 & $12 \%$ & 7 & $14 \%$ \\
\hline & Bachelor's & 28 & $56 \%$ & 27 & $54 \%$ \\
\hline & Master's & 9 & $18 \%$ & 10 & $20 \%$ \\
\hline & Doctoral & 1 & $2 \%$ & 0 & $0 \%$ \\
\hline \multicolumn{6}{|c|}{ Meditation Experience } \\
\hline & $<1$ time/week & 23 & $46 \%$ & 25 & $50 \%$ \\
\hline & 1-2 time/weeks & 16 & $32 \%$ & 13 & $26 \%$ \\
\hline & 3-4 time/weeks & 4 & $8 \%$ & 6 & $12 \%$ \\
\hline & 4-5 time/weeks & 3 & $6 \%$ & 1 & $2 \%$ \\
\hline & 6 + time/weeks & 4 & $8 \%$ & 5 & $10 \%$ \\
\hline \multicolumn{6}{|c|}{ Mental Health Conditions } \\
\hline & None & 33 & $65 \%$ & 27 & $57 \%$ \\
\hline & ADHD & 2 & $4 \%$ & 2 & $4 \%$ \\
\hline & Anxiety & 13 & $28 \%$ & 15 & $32 \%$ \\
\hline & Depression & 11 & $24 \%$ & 13 & $26 \%$ \\
\hline & Insomnia & 3 & $6 \%$ & 4 & $8 \%$ \\
\hline & PTSD & 1 & $2 \%$ & 2 & $4 \%$ \\
\hline
\end{tabular}

${ }^{a}$ Std Dev- Standard Deviation

their EEG patterns were shifting away from what typically coincides with a state of mental relaxation experience. In the current study, participants were encouraged to notice the brainwave patterns at the bottom of the screen and see if they could use their feelings of calm to keep the brainwave patterns above the threshold line. This feedback served as a gentle reminder to return their attention to the beach scene and the body scan meditation. The creators of the Healium experience noted that some first time users experience increased stress when the neurofeedback signal indicates a lack of success (S. Hill, personal communication, 18/Aug/ 2020). To minimize this potential impact, the threshold for the neurofeedback was set on "easy" to maximize success.

\section{Audio-Only Meditation Condition}

For the control condition, participants were fitted with over the ear headphones and given an iPad to listen to the audio track. The audio track was the audio used in the VR experience. The track was played for 1 minute while volume adjustments were made. Participants were then instructed to sit quietly and follow along with the guided meditation on the track for $5 \mathrm{~min}$.
TABLE 2 | Means and standard error estimates of BRUMS questionnaire measures.

\begin{tabular}{|c|c|c|c|c|c|}
\hline \multirow[t]{2}{*}{ Subscale } & \multirow[t]{2}{*}{ Group } & \multicolumn{2}{|c|}{ Pre } & \multicolumn{2}{|c|}{ Post } \\
\hline & & Mean & $\pm 95 \% \mathrm{Cl}^{\mathrm{a}}$ & Mean & $\pm 95 \% \mathrm{Cl}$ \\
\hline \multicolumn{6}{|l|}{ Anger } \\
\hline & Audio & 2.5 & 0.3 & 1.4 & 0.2 \\
\hline & VR & 0.9 & 0.2 & 0.1 & 0.1 \\
\hline \multicolumn{6}{|l|}{ Depression } \\
\hline & Audio & 2.4 & 0.3 & 0.8 & 0.2 \\
\hline & VR & 1.9 & 0.3 & 0.6 & 0.2 \\
\hline \multicolumn{6}{|l|}{ Tension } \\
\hline & Audio & 3.2 & 0.4 & 0.8 & 0.2 \\
\hline & VR & 2.6 & 0.4 & 0.74 & 0.3 \\
\hline \multicolumn{6}{|l|}{ Fatigue } \\
\hline & Audio & 5.5 & 0.4 & 6.0 & 0.5 \\
\hline & VR & 4.6 & 0.6 & 2.9 & 0.4 \\
\hline \multicolumn{6}{|l|}{ Vigor } \\
\hline & Audio & 5.3 & 0.5 & 6.2 & 0.4 \\
\hline & $\mathrm{VR}$ & 9.8 & 0.5 & 8.1 & 0.5 \\
\hline \multicolumn{6}{|l|}{ Happiness } \\
\hline & Audio & 9.1 & 0.4 & 9.0 & 0.4 \\
\hline & VR & 8.5 & 0.5 & 10.5 & 0.5 \\
\hline \multicolumn{6}{|l|}{ Calmness } \\
\hline & Audio & 8.6 & 0.4 & 8.0 & 0.6 \\
\hline & VR & 7.1 & 0.5 & 10.5 & 0.5 \\
\hline \multicolumn{6}{|l|}{ Confusion } \\
\hline & Audio & 2.1 & 0.3 & 2.2 & 0.2 \\
\hline & VR & 1.3 & 0.3 & 0.5 & 0.2 \\
\hline
\end{tabular}

${ }^{a} 95 \%$ Confidence Interval margins computed with Standard Error the Means for each group and subscale.

\section{Statistical Analysis}

Statistical analyses utilized Jamovi statistical software (Şahin and Aybek, 2019). Independent t-test were used to test for equivalency between groups prior to the VR experience, and on demographic and primary dependent measures. Mixed factorial ANOVA was used to examine group differences, main effects, and interactions of all experimental interventions $(\alpha=0.05)$. A Levene's test to examine the assumption of homogeneity of variance and Bonferroni Shapiro-Wilks test $(p<0.05)$ to test normality. The Bonferroni method was used to correct for multiple comparisons for Brunel subscales (Holm, 1979).

\section{RESULTS}

\section{User Statistics}

One hundred participants completed the study, with 50 in each group. The demographic information for each assigned group can be viewed in Table 1. Of the total sample, 91 identified as female and 9 identified as male. The average age of participants in the control group was 40.9 years old $(S D=13.90)$, and VR group was 42.6 years old $(S D=14.40)$. An independent samples t-test revealed no significant difference in mean age between groups $t 98=-0.44, p$ $=0.657$. Ninety-four percent of the sample were white, $3 \%$ identified as Asian American and 1\% identified in each of the following race/ ethnic categories: African American, Hispanic, and Middle Eastern. Level of education was very similar between groups with most participants having a Bachelor's degree. Both groups reported 


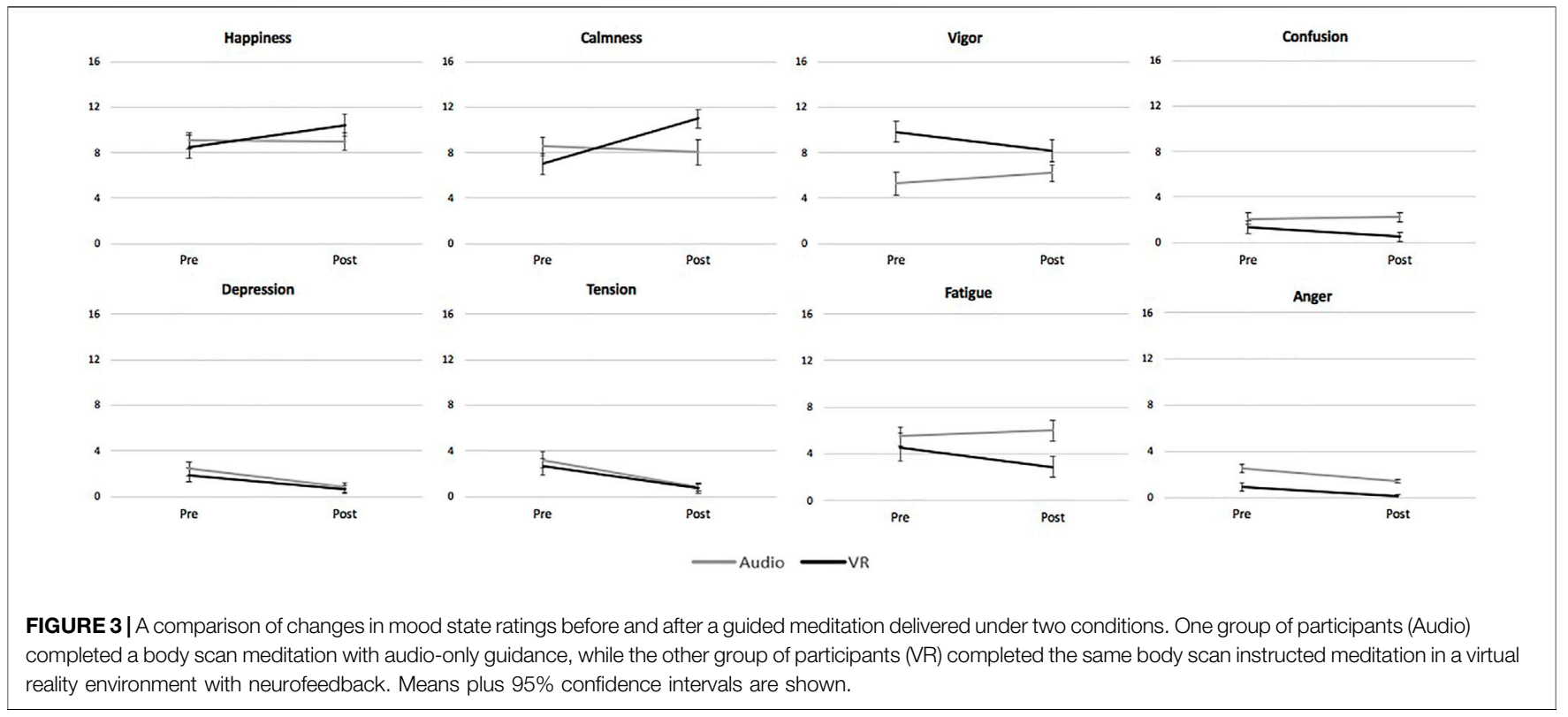

engaging in meditation a similar amount of time. The majority of participants in both groups meditated less than one time per week (control group $=47 \%$; VR group $=50 \%$ ). Mental health conditions were also similar between groups with the majority of each group having no known condition (control group $=57 \%$, VR group $=65 \%$ ) then anxiety (control group $=32 \%$, VR group $=28 \%$ ) and depression $($ control group $=26 \%$, VR group $=24 \%)($ Table 1$)$.

\section{Brunel Mood Scale}

A Cronbach Alpha test of each Brunel scale indicated acceptable internal consistency levels: Fatigue (Cronbach $\alpha=0.91$ ), Happiness (Cronbach $\alpha=0.85$ ), Calmness (Cronbach $\alpha=0.78$ ), Vigor (Cronbach $\alpha=0.79$ ), Tension (Cronbach $\alpha=0.78$ ), Depression (Cronbach $\alpha=0.76$ ), Anger (Cronbach $\alpha=0.76$ ), and Confusion (Cronbach $a=0.73$ )

While the general level of statistical significance was set at $p<0.05$, this value was adjusted for 8 comparisons based on a Bonferroni correction. The adjusted level of significance is $p<0.006(0.05 / 8)$. The results for each of the 8 subscales are reported below. Table 2 displays the pre and post group means for Brunel Mood ratings.

Significant group-by-time interactions were observed for Happiness $\left(\mathrm{F} 1,98=21.50, p<0.001, \eta p^{2}=0.18\right)$ and Calmness (F 1,98 = 46.1, $\left.p<0.001, \eta \mathrm{p}^{2}=0.32\right)$, as well as Vigor $(\mathrm{F} 1,98$ $\left.=21.05, p<0.001, \eta p^{2}=0.18\right)$, Fatigue $(\mathrm{F} 1,98=10.61$, $\left.p=0.002, \eta \mathrm{p}^{2}=0.10\right)$ and Confusion $(\mathrm{F} 1,98=9.02$, $\left.p=0.003, \eta p^{2}=0.08\right)$ subscales. On average, for Happiness the VR group endorsed higher scores $(\mathrm{M}$ dif $=+1.9, \mathrm{SE}=0.31)$ after their meditation, with a similar change not observed in the audio-only group ( $\mathrm{M}$ dif $=-0.1, \mathrm{SE}=0.31$ ). The VR group endorsed a moderate increase in Calmness ratings ( $M$ dif $=+4.0, \mathrm{SE}=0.36)$, while the audio only group showed a marginal decrease $(\mathrm{M}$ dif $=-0.5, \mathrm{SE}=0.57)$. For Confusion, on average, the VR group endorsed small decreases ( $\mathrm{M}$ dif $=-0.8$, $\mathrm{SE}=0.2$ ) while the audio-only group showed no reliable change $(\mathrm{M}$ dif $=+0.1, \mathrm{SE}=0.24)$. Fatigue ratings slightly decreased for the VR group ( $\mathrm{M}$ dif $=-1.7, \mathrm{SE}=0.45)$, and the audio-only group endorsed a small increase $(\mathrm{M}$ dif $=+0.5 \mathrm{SE}$ $=0.48)$. For Vigor ratings, the VR group scores decreased $(\mathrm{M}$ dif $=-1.7, \mathrm{SE}=0.43)$, and the audio-only group indicated a slight increase on average $(\mathrm{M}$ dif $=0.94, \mathrm{SE}=0.38)$. No significant group-by-time interactions emerged for Anger ( $\mathrm{F}$ $\left.1,98=1.24, p=0.268, \eta p^{2}=0.01\right)$ Tension $(\mathrm{F} 1,98=1.35$, $\left.p=0.248, \eta p^{2}=0.01\right)$ or Depression $(\mathrm{F} 1,98=1.09, p=0.299$, $\left.\eta p^{2}=0.01\right)$. Means and standard errors are reported in Table 2. Graphs of interactions are also shown in Figure 3.

Significant main effects for time were observed on the Tension $\left(\mathrm{F} 1,98=107.30, p<0.001, \eta \mathrm{p}^{2}=0.52\right)$ and Depression $(\mathrm{F} 1,98=$ 71.64, $\left.p<0.001, \eta p^{2}=0.42\right)$ mood scales. Taken together, both groups reported decreased Tension $(\mathrm{M}$ dif $=-2.1, \mathrm{SE}=0.2)$ and Depression $(\mathrm{M}$ dif $=-1.5, \mathrm{SE}=0.2)$ scores after the meditation. A main effect for time was also observed for Happiness ( $F$ 1,98 $\left.=17.5, p<0.001, \eta p^{2}=0.15\right)(\mathrm{M}$ dif $=+0.9, \mathrm{SE}=0.31)$ and Calmness $\left(\mathrm{F} 1,98=26.9, p<.001, \eta \mathrm{p}^{2}=0.22\right)$. $(\mathrm{M}$ dif $=+1.8, \mathrm{SE}$ $=0.34$ ) such that both groups reported an increase in scores from pre to post on both scales. A significant between group effect for Anger was observed (F 1,98 $\left.=40.4, p<.001, \eta p^{2}=0.29\right)$. The audio-only group rated higher on average than the $\mathrm{VR}+\mathrm{NF}$ group across both time points.

A significant main effect for time (F 1,98 = 39.21, $p<0.001$, $\left.\eta \mathrm{p}^{2}=0.29\right)$ was also detected for the Anger scale, signifying an overall incremental decrease for both groups $(\mathrm{M}$ dif $=-0.9, \mathrm{SE}$ $=0.1$ ). Despite differences at baseline these reductions were comparable. Between group effects were additionally detected for Vigor $(\mathrm{M} \mathrm{dif}=+0.92, \mathrm{SE}=0.31)$, such that the VR group rated 
TABLE 3 | F statistics, $p$ and power values for BRUMS effects.

\begin{tabular}{|c|c|c|c|c|}
\hline Subscale & Effect & F1,98 & $\mathbf{p}$ & np2 \\
\hline \multicolumn{5}{|l|}{ Anger } \\
\hline & Group & 40.4 & $<0.001$ & 0.29 \\
\hline & Time & 39.21 & $<0.001$ & 0.29 \\
\hline & Group X Time & 1.24 & 0.268 & 0.01 \\
\hline \multicolumn{5}{|l|}{ Depression } \\
\hline & Group & 1.32 & 0.254 & 0.01 \\
\hline & Time & 71.64 & $<0.001$ & 0.42 \\
\hline & Group X Time & 1.09 & 0.299 & 0.01 \\
\hline \multicolumn{5}{|l|}{ Tension } \\
\hline & Group & 0.829 & 0.365 & 0.01 \\
\hline & Time & 107.30 & $<0.001$ & 0.52 \\
\hline & Group X Time & 1.35 & 0.248 & 0.01 \\
\hline \multicolumn{5}{|l|}{ Fatigue } \\
\hline & Group & 11.00 & 0.001 & 0.10 \\
\hline & Time & 3.23 & 0.076 & 0.03 \\
\hline & Group X Time & 10.61 & 0.002 & 0.10 \\
\hline \multicolumn{5}{|l|}{ Vigor } \\
\hline & Group & 30.70 & $<0.001$ & 0.24 \\
\hline & Time & 1.74 & 0.190 & 0.02 \\
\hline & Group X Time & 21.05 & $<0.001$ & 0.18 \\
\hline \multicolumn{5}{|l|}{ Happiness } \\
\hline & Group & 0.69 & 0.408 & 0.01 \\
\hline & Time & 17.5 & $<0.001$ & 0.15 \\
\hline & Group X Time & 21.50 & $<0.001$ & 0.18 \\
\hline \multicolumn{5}{|l|}{ Calmness } \\
\hline & Group & 1.96 & 0.164 & 0.02 \\
\hline & Time & 26.9 & $<0.001$ & 0.22 \\
\hline & Group X Time & 46.1 & $<0.001$ & 0.32 \\
\hline \multicolumn{5}{|l|}{ Confusion } \\
\hline & Group & 16.1 & $<0.001$ & 0.14 \\
\hline & Time & 4.45 & 0.038 & 0.04 \\
\hline & Group X Time & 9.02 & 0.003 & 0.08 \\
\hline
\end{tabular}

themselves higher in vigor on average than the audio only group. No group-level main effects were observed across the remaining scales (p's > 0.05). Statistics for all effects are reported in Table 3.

\section{DISCUSSION}

In this study we examined the feasibility and comparative mood effects of a brief immersive VR meditation with NF, to an audioonly guided meditation in front-line healthcare workers during shift work in a hospital setting. It was hypothesized that both groups would show decreases in negative mood states and increases in positive mood states after a brief guided body scan meditation. Furthermore, it was hypothesized that the $\mathrm{VR}+\mathrm{NF}$ delivery would generally increase these effects, by creating a more engaging and immersive meditation environment. Extended or longer-term impacts of such an intervention were not evaluated in this study.

Overall, the VR+NF delivery resulted in parallel or enhanced effects across the various aspects of mood experiences evaluated. On average, both groups showed changes in mood profiles generally in line with an overall improved mood state. Across a select few mood scales, the VR+NF delivery produced improved outcomes relative to the changes in the audio-only group. There was no evidence that the addition of $\mathrm{VR}+\mathrm{NF}$ to the guided meditation led to any significant negative outcomes relative to the audio-only condition, or in general. Participants were queried regarding any adverse experiences and none were reported. This finding may be important as other research with VR has noted common side effects including simulation sickness or cyber sickness (Nichols, and Patel, 2002; Botella, et al., 2009; Baniasadi, et al., 2020). The lack of adverse events in the current study may be related to the brief exposure as well as the fact that the experience was stationary. It is also noted that the inclusion of a visual neurofeedback signal could potentially create stress in some users. To minimize this effect, the default threshold for success was automatically set to maximize the amount of time users were meeting criterion. While stress related to the neurofeedback signal was not directly assessed, there were no instances of participants describing this concern during the study. Further, the positive shifts in mood observed in this group suggest that this was not a significant concern.

In terms of the similarities between the audio-only and $\mathrm{VR}+\mathrm{NF}$ effects, both groups demonstrated highly comparable decreases across Anger, Depression, and Tension ratings after completion of the meditation. In both cases the self-reported scores were relatively low to begin with, but the meditation still produced a reliable reduction in these negative mood domains across groups. This result supports the notion that even a brief guided meditation can alleviate mild negative mood symptoms. However, because of the floor effect associated with these low scores, it is unclear whether individuals with higher baselines of anger, depression, or tension would respond more or less strongly to this meditation in either delivery format. Regardless, given the brevity and potential repeatability across the workday, this still comes forward as a promising and worthwhile finding. This reduction in negative mood states seems particularly noteworthy as previous research with the BRUMS has demonstrated a more limited impact. When assessing the impact of a brief $(4.5 \mathrm{~min}) \mathrm{VR}$ meditation just prior to a needle stick at a blood drive, participants reported significant reductions in Tension and Fatigue, increased feelings of Calmness and Happiness, but no significant changes in Anger, Confusion, Depression, or Vigor (Tarrant et al., 2019). It is unclear why a similar intervention would have somewhat different impacts. However, the participants in the current study were facing a chronic stressor while those in the blood drive study were facing an acute stressor. In addition, the meditation in the current study was a body scan, while the meditation in the blood drive study was focused on enhancing feelings of gratitude. It is also noted that the current study had more participants, providing more statistical power. Clearly, more studies are needed to clarify the influence of meditation type, participant characteristics, type of stressor experienced, and sample size on such findings.

Significant between group interactions were found on each of the scales assessing positively valenced mood states, including Happiness, Calmness, and Vigor. While the audio-only condition showed almost no change on the Happiness and Calmness scales, the VR+NF group showed significant increases on both. Interestingly, the audio-only delivery increased Vigor, whereas the VR+NF meditation resulted in a drop in Vigor; however, both groups decreased in Fatigue ratings alongside these differences in 
Vigor ratings. Items on the Vigor scale include lively, energetic, active, and alert. The items on the Fatigue subscale include feeling worn out, exhausted, sleepy, and tired. This difference in trajectories on the Vigor subscale might best be accounted for alongside the significant boost in Calmness experienced by the $\mathrm{VR}+\mathrm{NF}$ condition, which was absent in the audio-only condition. In another study comparing two extended reality meditations with and without neurofeedback, a 4-min augmented reality (AR) guided meditation resulted in both groups showing increased ratings of Happiness and Calmness on the BRUMS as well as significant decreases in Depression, Tension, and Fatigue. No significant changes were observed on scales measuring Anger, Confusion, or Vigor and there were no significant differences between groups (Viczko et al., 2021). While AR is less engaging than VR, it seems important to note that the presence of neurofeedback did not significantly influence the self-report results of mood changes. This suggests that the positive influence was connected only to the AR meditation itself. It is not clear that this conclusion can extend to the current study, but does present a direction for future research in this area.

These results suggest that a brief, body scan guided meditation can be an effective tool for reducing negative mood states within a healthcare environment, regardless of the delivery methods explored in this study. Perhaps the reduction of negative mood states is a somewhat generic result of brief relaxation interventions. Supporting this idea, Tarrant et al. (2018) measured changes in state anxiety following a brief, eyes closed relaxation period, followed by either another relaxation period or a VR meditation experience. In this case, both groups reported a significant reduction of anxiety after the resting period and then a more dramatic decrease in anxiety after the VR experience. Similarly, Carissoli et al. (2015) compared an appbased mindfulness meditation to a relaxation music control group. Again, both groups demonstrated a significant reduction in stress. However, in other studies meditation practices have demonstrated unique effects that distinguish the outcomes of meditation as going beyond simple relaxation effects (Gillani and Smith 2001; Tang et al., 2009; Sedlmeier et al., 2012).

\section{Limitations and Future Directions}

When considering the positive mood domains where $\mathrm{VR}+\mathrm{NF}$ delivery outperformed the audio-only meditation, there are several possible factors which will need to be clarified in future studies. The VR+NF modality has a number of experiential components which may have led to increases in happiness and calmness above the audio-only meditation effects. How much of the increased effects were due to the virtual environment (e.g., increased level of presence; Graffigna et al., 2013; Flavián et al., 2020) versus the addition of neurofeedback guidance remains to be examined in future research. Additionally, novelty and expectation effects related to meditating with cutting edge technology may have also influenced the outcomes. In both cases, research designs that include yoked or sham/placebo experimental conditions could help to provide quantitative clarification regarding the amount of influence these factors play in driving user experiences and outcomes. Qualitative and phenomenological interviewing methods may also help to understand the role these factors play, and guide confirmatory experimental studies of this and similar technology assisted meditation programs.

The results from this study are consistent with previous research showing that meditation experiences can have a positive impact on mood states, with and without VR (Goyal et al., 2014). Whether concentrated training in meditation consistently with or without the addition of VR or NF technologies leads to further difference in training trajectories and outcomes remains to be tested in future studies. It is possible that the mood effects observed here could level out, converge, or increase in magnitude of effects as meditative proficiency increases with continuous training. It is also possible that changes in the neurofeedback thresholding could impact learning (Kadosh and Staunton, 2019). A limitation here was that we were not able to retrieve the EEG or other biometric data from participants as they practiced. Physiological measurements would be desirable in future studies to help delineate the effects of VR or NF on the mind and body, versus meditating without the addition of these technological aids.

Our focus here was on testing a brief intervention for supporting healthcare workers during a historically stressful period of time dealing with global pandemic and healthcare service strain. Despite the apparent work place stress, the participants in this study demonstrated overall low baseline ratings of Tension and Depression. As not all participants were assigned to COVID wards, it is possible that their levels of stress and burnout were less than those reported for frontline healthcare providers (De Hert, 2020; Orrù et al., 2021). A goal for future studies might include selecting healthcare workers who are reporting difficulties coping or higher than typical distress or burnout. Specifically, the current study examined a broad range of hospital employees, not only nurses assigned to COVID-19 wards. It is possible that nurses assigned specifically to COVID-19 wards, experiencing the most stress and burnout during the pandemic, would have responded differently to these interventions.

In addition, it is noted that the participants in this study were predominately White and female-identified. While there is limited research on burnout among Black, Indigenous, and persons of color (BIPOC) healthcare workers, recent attention to these groups suggests that they are likely to be vulnerable to increased levels of burnout and vicarious trauma (Greenberg, 2020; Miu and Moore, 2021). In addition to the emotional burden of caring for others in a time of uncertainty and fear, BIPOC providers also endure the myriad effects of structural racism, contributing to an increased risk of mental health concerns (American Psychiatric Association, 2017). Consequently, the results of the current study offer limited generalizability to these and other oppressed populations. Future studies should actively seek to include more diversity in their sample.

Future investigations could also be improved by the inclusion of a broader selection of targeted outcome measures, particularly for the healthcare worker population. This might include perceived stress measures, measures of self- or othercompassion, or workplace satisfaction measures. While we specifically chose a brief intervention for this study, it would also be important to examine longer meditations as well as other 
meditation techniques. Techniques such as compassion fatigue, loving-kindness and compassion-generation meditations may be of great utility.

As the data for this study was collected over a 6-month period within a single hospital, it is likely that some of the healthcare workers volunteering for the study were aware of the two different interventions prior to their participation. This awareness could have led to some of the audio-only participants being disappointed, less motivated, interested, or engaged, potentially influencing their responses to the intervention. Future studies should provide additional controls to ensure that the various conditions are not aware of the other experimental conditions. In addition, it would be helpful to include additional comparison groups, including wait list control, 2D and augmented reality meditations versus virtual reality, with or without biofeedback inputs, or whether patient versus staff have different experiences with meditation resources available on site. These areas of focus would help to reveal the specificity and generalizability of meditation interventions similar to the ones explored here.

\section{CONCLUSION}

In this study, we compared an audio-only body scan meditation to a virtual reality plus neurofeedback version. Specifically, we tested the impact of these interventions on mood states of healthcare workers in a hospital setting during the Covid-19 pandemic. Our results demonstrated that both delivery formats led to a significant and comparable decrease of certain negative mood states (Anger, Depression, Tension). Significant differences between interventions were observed on all other scales. The VR group increased scores on Happiness and Calmness while the audio-only group did not. The VR group demonstrated decreases in Confusion while the audio-only group reported slight increases. On the Fatigue scale, the VR group reported slight decreases, while the audio-only group indicated slight increases. On Vigor ratings, the VR group showed decreases in ratings while the audio-only group indicated slight increases. There were no adverse reactions noted in either condition. Overall, these results suggest that a brief, body scan guided meditation can be an effective tool for reducing negative mood states within a healthcare environment, regardless of the delivery method.

\section{REFERENCES}

Alharbi, J., Jackson, D., and Usher, K. (2020). The Potential for COVID-19 to Contribute to Compassion Fatigue in Critical Care Nurses. J. Clin. Nurs. 29, 2762-2764. doi:10.1111/jocn.15314

American Psychiatric Association (2017). Mental Health disparities:Diverse Populations. Available at: https://www.psychiatry.org/psychiatrists/ culturalcompetency/education/mental-health-facts.

Asmundson, G. J. G., and Taylor, S. (2020). How Health Anxiety Influences Responses to Viral Outbreaks like COVID-19: What All Decision-Makers, Health Authorities, and Health Care Professionals Need to Know. J. Anxiety Disord. 71, 102211. doi:10.1016/j.janxdis.2020.102211

Athanas, A. J., McCorrison, J. M., Smalley, S., Price, J., Grady, J., Wehner, P., et al. (2019). Association between Improvement in Baseline Mood and Long-Term
The addition of VR and neurofeedback to the meditation appears to provide additional gains in positive mood states. Future studies should delineate the specific influences contained within the VR+NFB condition as well as examine the utility as a longer-term intervention within healthcare settings.

\section{DATA AVAILABILITY STATEMENT}

The raw data supporting the conclusions of this article will be made available by the authors, without undue reservation.

\section{ETHICS STATEMENT}

The studies involving human participants were reviewed and approved by the Washington University Review Board (Columbia, Missouri, United States). The patients/participants provided their written informed consent to participate in this study.

\section{AUTHOR CONTRIBUTIONS}

JT contributed to conception, study design, and manuscript writing. RJ contributed to data organization, analyses and manuscript writing. JV contributed to data analyses and manuscript writing.

\section{FUNDING}

GSK Consumer Healthcare.

\section{ACKNOWLEDGMENTS}

Mihaela Popescu and Michele Sprague contributed to IRB authorization, participant recruitment, and on-site data collection at Boone Hospital Center, Columbia, Missouri.

Use of a Mindfulness and Meditation App: Observational Study. JMIR Ment. Health 6 (5), e12617. doi:10.2196/12617

Baniasadi, T., Ayyoubzadeh, S. M., and Mohammadzadeh, N. (2020). Challenges and Practical Considerations in Applying Virtual Reality in Medical Education and Treatment. Oman Med.J. 35 (3), e125. doi:10.5001/ omj.2020.43

Bao, Y., Sun, Y., Meng, S., Shi, J., and Lu, L. (2020). 2019-nCoV Epidemic: Address Mental Health Care to Empower Society. The Lancet 395 (10224), e37-e38. doi:10.1016/S0140-6736(20)30309-3

Berger, A. M., and Davelaar, E. J. (2018). Frontal Alpha Oscillations and Attentional Control: A Virtual Reality Neurofeedback Study. Neuroscience 378 (15), 189-197. doi:10.1016/j.neuroscience.2017.06.007

Botella, A. C., García Palacios, A., Baños Rivera, R. M., and Quero Castellano, S. (2009). Cybertherapy: Advantages, Limitations, and Ethical Issues. Psychnology J. 7 (1), 77-100. doi:10.5944/ap.6.1.447 
Brandmeyer, T., and Delorme, A. (2020). Closed-Loop Frontal Midline $\theta$ Neurofeedback: A Novel Approach for Training Focused-Attention Meditation. Front. Hum. Neurosci. 14, 246. doi:10.3389/fnhum.2020. 00246

Brandmeyer, T., and Delorme, A. (2013). Meditation and Neurofeedback. Front. Psychol. 4, 688. doi:10.3389/fpsyg.2013.00688

Çakiroğlu, A. A., Demir, E., and Güçlü, M. (2020). The Validity and Reliability Study of the Brunel Mood Scale with the Adult Athletes (Turkish Adaptation). Int. J. Appl. Exerc. Physiol. 9 (10), 126-140. doi:10.17220/ ijpes.2014.01.001

Carissoli, C., Villani, D., and Riva, G. (2015). Does a Meditation Protocol Supported by a mobile Application Help People Reduce Stress? Suggestions from a Controlled Pragmatic Trial. Cyberpsychology, Behav. Soc. Networking 18 (1), 46-53. doi:10.1089/cyber.2014.0062

Carl, E., Stein, A. T., Levihn-Coon, A., Pogue, J. R., Rothbaum, B., Emmelkamp, P., et al. (2019). Virtual Reality Exposure Therapy for Anxiety and Related Disorders: A Meta-Analysis of Randomized Controlled Trials. J. anxiety Disord. 61, 27-36. doi:10.1016/j.janxdis.2018.08.003

Chirico, F., Nucera, G., and Magnavita, N. (2021). Protecting the Mental Health of Healthcare Workers during the COVID-19 Emergency. Bjpsych Int. 18 (1), 1-2. doi:10.1192/bji.2020.39

Coulon, S. M., Monroe, C. M., and West, D. S. (2016). A Systematic, MultiDomain Review of mobile Smartphone Apps for Evidence-Based Stress Management. Am. J. Prev. Med. 51 (1), 95-105. doi:10.1016/j.amepre.2016. 01.026

Cullen, W., Gulati, G., and Kelly, B. D. (2020). Mental Health in the Covid-19 Pandemic. QJM: Int. J. Med. 113, 311-312. doi:10.1093/qjmed/hcaal10

De Hert, S. (2020). Burnout in Healthcare Workers: Prevalence, Impact and Preventative Strategies. Lra 13, 171-183. doi:10.2147/lra.s240564

Döllinger, N., Wienrich, C., and Latoschik, M. E. (2021). Challenges and Opportunities of Immersive Technologies for Mindfulness Meditation: A Systematic Review. Front. Virtual Real. 2, 29. doi:10.3389/frvir.2021. 644683

Eichenberg, C., and Wolters, C. (2012). Virtual Reality in Psychological, Medical and Pedagogical Applications, 2. London, UK: IntechOpen, 35-64. doi:10.5772/ 2607

Evans, S., Wyka, K., Blaha, K. T., and Allen, E. S. (2018). Self-compassion Mediates Improvement in Well-Being in a Mindfulness-Based Stress Reduction Program in a Community-Based Sample. Mindfulness 9 (4), 1280-1287. doi:10.1007/ s12671-017-0872-1

Faul, F., Erdfelder, E., Lang, A.-G., and Buchner, A. (2007). G*Power 3: A Flexible Statistical Power Analysis Program for the Social, Behavioral, and Biomedical Sciences. Behav. Res. Methods 39 (2), 175-191. doi:10.3758/ bf03193146

Fergusson, D. M., Horwood, L. J., Boden, J. M., and Mulder, R. T. (2014). Impact of a Major Disaster on the Mental Health of a Well-Studied Cohort. JAMA psychiatry 71 (9), 1025-1031. doi:10.1001/ jamapsychiatry.2014.652

Flavián, C., Ibáñez-Sánchez, S., and Orús, C. (2021). The Influence of Scent on Virtual Reality Experiences: the Role of Aroma-Content Congruence. J. Business Res. 123, 289-301. doi:10.1016/j.jbusres.2020.09.036

Flett, J. A. M., Hayne, H., Riordan, B. C., Thompson, L. M., and Conner, T. S. (2019). Mobile Mindfulness Meditation: a Randomised Controlled Trial of the Effect of Two Popular Apps on Mental Health. Mindfulness 10 (5), 863-876. doi:10.1007/s12671-018-1050-9

Fox, K. C. R., Dixon, M. L., Nijeboer, S., Girn, M., Floman, J. L., Lifshitz, M., et al. (2016). Functional Neuroanatomy of Meditation: A Review and Meta-Analysis of 78 Functional Neuroimaging Investigations. Neurosci. Biobehavioral Rev. 65, 208-228. doi:10.1016/j.neubiorev.2016.03.021

Freeman, D., Reeve, S., Robinson, A., Ehlers, A., Clark, D., Spanlang, B., et al. (2017). Virtual Reality in the Assessment, Understanding, and Treatment of Mental Health Disorders. Psychol. Med. 47 (14), 2393-2400. doi:10.1017/ s003329171700040x

Freitas, J. R. S., Velosa, V. H. S., Abreu, L. T. N., Jardim, R. L., Santos, J. A. V., Peres, B., et al. (2021). Virtual Reality Exposure Treatment in Phobias: a Systematic Review. Psychiatr. Q. 92, 1685-1710. doi:10.1007/s11126-02109935-6
Garrison, K. A., Santoyo, J. F., Davis, J. H., Thornhill, T. A., Kerr, C. E., and Brewer, J. A. (2013). Effortless Awareness: Using Real Time Neurofeedback to Investigate Correlates of Posterior Cingulate Cortex Activity in Meditators' Self-Report. Front. Hum. Neurosci. 7, 440. doi:10.3389/ fnhum.2013.00440

Gillani, N. B., and Smith, J. C. (2001). Zen Meditation and ABC Relaxation Theory: An Exploration of Relaxation States, Beliefs, Dispositions, and Motivations. J. Clin. Psychol. 57 (6), 839-846. doi:10.1002/jclp.1053

Gorini, A., Pallavicini, F., Algeri, D., Repetto, C., Gaggioli, A., and Riva, G. (2010). Virtual Reality in the Treatment of Generalized Anxiety Disorders. Stud. Health Technol. Inform. 154, 39-43. doi:10.1586/14737175.8.2.215

Goyal, M., Singh, S., Sibinga, E. M. S., Gould, N. F., Rowland-Seymour, A., Sharma, R., et al. (2014). Meditation Programs for Psychological Stress and Well-Being: a Systematic Review and Meta-Analysis. JAMA Intern. Med. 174 (3), 357-368. doi:10.1001/jamainternmed.2013.13018

Graffigna, G., Barello, S., and Riva, G. (2013). How to Make Health Information Technology Effective: The Challenge of Patient Engagement. Arch. Phys. Med. Rehabil. 94, 2034-2035. doi:10.1016/j.apmr.2013.04.024

Greenberg, T. M. (2020). "Vicarious Trauma and Self-Care for the Trauma Therapist," in Treating Complex Trauma (Cham: Springer), 191-211. doi:10. 1007/978-3-030-45285-8_10

Hammond, D. (2005). Neurofeedback with Anxiety and Affective Disorders. Child Adolesc. Psychiatr. Clin. North America 14, 105-123. doi:10.1016/j.chc.2004. 07.008

Holm, S. (1979). A Simple Sequentially Rejective Multiple Test Procedure. Scand. J. Stat. 6 (2), 65-70.

Kabat-Zinn, J. (2003). Mindfulness-based Interventions in Context: Past, Present, and Future. Clin. Psychol. Sci. Pract. 10, 144-156. doi:10.1093/ clipsy.bpg016

Kadosh, K. C., and Staunton, G. (2019). A Systematic Review of the Psychological Factors that Influence Neurofeedback Learning Outcomes. NeuroImage 185 (15), 545-555. doi:10.1016/j.neuroimage.2018.10.021

Krystal, J. H., and McNeil, R. L. (2020). Responding to the Hidden Pandemic for Healthcare Workers: Stress. Nat. Med. 26, 639. doi:10.1038/s41591-020$0878-4$

Liu, J. H., Wang, W., and Gao, W. B. (2004). Study on Effect of SARS on Mental Health of Medical Staffs in Fever Clinic of Military Hospital. Nanfang J. Nurs. 2004, 9-10. doi:10.1177/2F070674370905400504

Liu, S., Yang, L., Zhang, C., Xiang, Y.-T., Liu, Z., Hu, S., et al. (2020). Online Mental Health Services in China during the COVID-19 Outbreak. The Lancet Psychiatry 7 (4), e17-e18. doi:10.1016/S22150366(20)30077-8

Malbos, E., Boyer, L., and Lançon, C. (2013). L'utilisation de la réalité virtuelle dans le traitement des troubles mentaux. La Presse Médicale 42, 1442-1452. doi:10. 1016/j.lpm.2013.01.065

Maples-Keller, J. L., Bunnell, B. E., Kim, S.-J., and Rothbaum, B. O. (2017). The Use of Virtual Reality Technology in the Treatment of Anxiety and Other Psychiatric Disorders. Harv. Rev. Psychiatry 25 (3), 103-113. doi:10.1097/ hrp. 0000000000000138

Miu, A. S., and Moore, J. R. (2021). Behind the Masks: Experiences of Mental Health Practitioners of Color during the COVID-19 Pandemic. Acad. Psychiatry 45, 539-544. doi:10.1007/s40596-021-01427-w

Morina, N., Ijntema, H., Meyerbröker, K., and Emmelkamp, P. M. G. (2015). Can Virtual Reality Exposure Therapy Gains Be Generalized to Real-Life? A MetaAnalysis of Studies Applying Behavioral Assessments. Behav. Res. Ther. 74, 18-24. doi:10.1016/j.brat.2015.08.010

Motraghi, T. E., Seim, R. W., Meyer, E. C., and Morissette, S. B. (2014). Virtual Reality Exposure Therapy for the Treatment of Posttraumatic Stress Disorder: a Methodological Review Using CONSORT Guidelines. J. Clin. Psychol. 70 (3), 197-208. doi:10.1002/jclp.22051

Nica, E. (2017). Political Mendacity and Social Trust. Educ. Philos. Theor. 49 (6), 571-572. doi:10.1080/00131857.2017.1288787

Nichols, S., and Patel, H. (2002). Health and Safety Implications of Virtual Reality: a Review of Empirical Evidence. Appl. Ergon. 33 (3), 251-271. doi:10.1016/ S0003-6870(02)00020-0

Olbrich, S., Sander, C., Matschinger, H., Mergl, R., Trenner, M., Schönknecht, P., et al. (2011). Brain and Body: Associations between EEG-Vigilance and the 
Autonomous Nervous System Activity during Rest. J. Psychophysiology 25, 190-200. doi:10.1027/0269-8803/a000061

Orakpo, N., Vieux, U., and Castro-Nuñez, C. (2021). Case Report: Virtual Reality Neurofeedback Therapy as a Novel Modality for Sustained Analgesia in Centralized Pain Syndromes. Front. Psychiatry 12, 480. doi:10.3389/fpsyt. 2021.660105

Orrù, G., Marzetti, F., Conversano, C., Vagheggini, G., Miccoli, M., Ciacchini, R., et al. (2021). Secondary Traumatic Stress and Burnout in Healthcare Workers during COVID-19 Outbreak. Ijerph 18 (1), 337. doi:10.3390/ ijerph 18010337

Philippot, P., Nef, F., Clauw, L., Romrée, M., and Segal, Z. (2012). A Randomized Controlled Trial of Mindfulness-Based Cognitive Therapy for Treating Tinnitus. Clin. Psychol. Psychother. 19, 411-419. doi:10.1002/ cpp.756

Price, J., and Budzynski, T. (2009). "Anxiety, EEG Patterns, and Neurofeedback," in Introduction to Quantitative EEG and Neurofeedback: Advanced Theory and Applications (Cambridge, USA: Academic Press), 453-472. doi:10.1016/b9780-12-374534-7.00017-4

Richardson, K. M., and Rothstein, H. R. (2008). Effects of Occupational Stress Management Intervention Programs: a Meta-Analysis. J. Occup. Health Psychol. 13 (1), 69-93. doi:10.1037/1076-8998.13.1.69

Roquet, C. D., and Sas, C. (2018). "Evaluating Mindfulness Meditation Apps," in Extended Abstracts of the 2018 CHI Conference on Human Factors in Computing Systems (CHI EA '18) (New York, NY, USA: Association for Computing Machinery), 1-6. doi:10.1145/3170427.3188616

Rus-Calafell, M., Garety, P., Sason, E., Craig, T. J. K., and Valmaggia, L. R. (2018). Virtual Reality in the Assessment and Treatment of Psychosis: a Systematic Review of its Utility, Acceptability and Effectiveness. Psychol. Med. 48 (3), 362-391. doi:10.1017/s0033291717001945

Ruths, F., and Thompson, L. (2009). "Principles and Practice of Stress Management (3rd Edn). Edited by Paul M. Lehrer, Robert L. Woolfolk and Wesley E. Sime Guilford Press. 2007. 721 1pp. US\$85.00 (Hb). ISBN: 9781593850005 ," in Principles and Practice of Stress Management. Editors P. M. Lehrer, R. L. Woolfolk, and W. E. Sime. 3rd Edn (New York, NY: Guilford Publications), 194, 93. doi:10.1192/ bjp.bp.107.047134

Şahin, M., and Aybek, E. (2019). Jamovi: an Easy to Use Statistical Software for the Social Scientists. Int. J. Assess. Tools Edu. 6 (4), 670-692. doi:10.21449/ijate. 661803

Sedlmeier, P., Eberth, J., Schwarz, M., Zimmermann, D., Haarig, F., Jaeger, S., et al. (2012). The Psychological Effects of Meditation: a Meta-Analysis. Psychol. Bull. 138 (6), 1139-1171. doi:10.1037/a0028168

Sherlin, L., Gevirtz, R., Wyckoff, S., and Muench, F. (2009). Effects of Respiratory Sinus Arrhythmia Biofeedback versus Passive Biofeedback Control. Int. J. Stress Manage. 16 (3), 233-248. doi:10.1037/a0016047

Sherlin, L., Muench, F., and Wyckoff, S. (2010). Respiratory Sinus Arrhythmia Feedback in a Stressed Population Exposed to a Brief Stressor Demonstrated by Quantitative EEG and sLORETA. Appl. Psychophysiol Biofeedback 35 (3), 219-228. doi:10.1007/s10484-010-9132-z

Slater, M., and Wilbur, S. (1997). A Framework for Immersive Virtual Environments (Five): Speculations on the Role of Presence in Virtual Environments. Presence: Teleoperators \& Virtual Environments 6, 603-616. doi:10.1162/pres.1997.6.6.603

Smith, M. L., Collura, T. F., and Tarrant, J. (2014). "Neurofeedback," in EEG/ERP Analysis: Methods and Applications. Editors K. Nidal and A. S. Malik (Crc Press), 184-190. doi:10.4324/9780203795132-10

Soos, I., Lane, A. M., Leibinger, E., Karsai, I., and Hamar, P. (2007). Validity of the Brunel Mood Scale for Use with UK. Italian and Hungarian Athletes. J. Sports Sci. Med. 11 (1), 119-130. doi:10.1080/1612197x.2021.1919740

Tang, Y.-Y., Ma, Y., Fan, Y., Feng, H., Wang, J., Feng, S., et al. (2009). Central and Autonomic Nervous System Interaction Is Altered by Short-Term Meditation. Proc. Natl. Acad. Sci. 106 (22), 8865-8870. doi:10.1073/pnas.0904031106

Tarrant, J., Abrams, J. S., and Jackson, R. (2019). The Impact of Virtual Reality on Mood States Prior to Blood Donation. Sjpbs 2 (5), 19-20. doi:10.32474/SJPBS. 2019.02.000150

Tarrant, J., and Cope, H. (2018). Combining Frontal Gamma Asymmetry Neurofeedback with Virtual Reality: A Proof of Concept Case Study. $\mathrm{Nr} 5$ (2), 57-66. doi:10.15540/nr.5.2.57
Tarrant, J. (2017). Meditation Interventions to Rewire the Brain: Integrating Neuroscience Strategies for ADHD, Anxiety, Depression and PTSD. Eau Claire, WI: PESI Publishing and Media.

Tarrant, J., Viczko, J., and Cope, H. (2018). Virtual Reality for Anxiety Reduction Demonstrated by Quantitative EEG: a Pilot Study. Front. Psychol. 9, 1280. doi:10.3389/fpsyg.2018.01280

Taylor, S., Landry, C. A., Paluszek, M. M., and Asmundson, G. J. G. (2020). Reactions to COVID-19: Differential Predictors of Distress, Avoidance, and Disregard for Social Distancing. J. Affective Disord. 277, 94-98. doi:10.1016/j.jad.2020.08.002

Teasdale, J. D., Segal, Z. V., Williams, J. M. G., Ridgeway, V. A., Soulsby, J. M., and Lau, M. A. (2000). Prevention of Relapse/recurrence in Major Depression by Mindfulness-Based Cognitive Therapy. J. Consulting Clin. Psychol. 68, 615-623. doi:10.1037/0022-006x.68.4.615

Travis, F., and Shear, J. (2010). Focused Attention, Open Monitoring and Automatic Self-Transcending: Categories to Organize Meditations from Vedic, Buddhist and Chinese Traditions. Conscious. Cogn. 19 (4), 1110-1118. doi:10.1016/j.concog.2010.01.007

Tussyadiah, I. P., Wang, D., Jung, T. H., and tom Dieck, M. C. (2018). Virtual Reality, Presence, and Attitude Change: Empirical Evidence from Tourism. Tourism Manage. 66, 140-154. doi:10.1016/j.tourman.2017.12.003

Valmaggia, L. R., Latif, L., Kempton, M. J., and Rus-Calafell, M. (2016). Virtual Reality in the Psychological Treatment for Mental Health Problems: An Systematic Review of Recent Evidence. Psychiatry Res. 236, 189-195. doi:10. 1016/j.psychres.2016.01.015

Van Lutterveld, R., Houlihan, S. D., Pal, P., Sacchet, M. D., McFarlane-Blake, C., Patel, P. R., et al. (2017). Source-space EEG Neurofeedback Links Subjective Experience with Brain Activity during Effortless Awareness Meditation. Neuroimage 151, 117-127. doi:10.1016/j.neuroimage.2016.02.047

Veling, W., Brinkman, W.-P., Dorrestijn, E., and Van Der Gaag, M. (2014). Virtual Reality Experiments Linking Social Environment and Psychosis: a Pilot Study. Cyberpsychology, Behav. Soc. Networking 17 (3), 191-195. doi:10.1089/cyber. 2012.0497

Viczko, J., Tarrant, J., and Jackson, R. (2021). Effects on Mood and EEG States after Meditation in Augmented Reality with and without Adjunctive Neurofeedback. Front. Virtual Real. 2, 9. doi:10.3389/frvir.2021.618381

Wang, J., Cheng, Y., Zhou, Z., Jiang, A., Guo, J., Chen, Z., et al. (2020). Psychological Status of Wuhan Medical Staff in Fighting against COVID-19. Med. J. Wuhan Univ. 4, 547-550. doi:10.14188/j.1671-8852.2020.0098

Wang, X., Gao, L., Shinfuku, N., Zhang, H., Zhao, C., and Shen, Y. (2000). Longitudinal Study of Earthquake-Related PTSD in a Randomly Selected Community Sample in north China. Ajp 157 (8), 1260-1266. doi:10.1176/ appi.ajp.157.8.1260

Williams, J. M. G., Duggan, D. S., Crane, C., and Fennell, M. J. V. (2006). Mindfulness-based Cognitive Therapy for Prevention of Recurrence of Suicidal Behavior. J. Clin. Psychol. 62, 201-210. doi:10.1002/jclp. 20223

Yang, E., Schamber, E., Meyer, R. M. L., and Gold, J. I. (2018). Happier Healers: Randomized Controlled Trial of mobile Mindfulness for Stress Management. J. Altern. Complement. Med. 24 (5), 505-513. doi:10.1089/acm.2015.0301

Conflict of Interest: JT is contracted by Healium XR to assist in product development and assessment. Coauthors JV and RJ, are not affiliated with Healium XR, were recruited to conduct statistical analyses and assist in the interpretation of this data.

Publisher's Note: All claims expressed in this article are solely those of the authors and do not necessarily represent those of their affiliated organizations, or those of the publisher, the editors and the reviewers. Any product that may be evaluated in this article, or claim that may be made by its manufacturer, is not guaranteed or endorsed by the publisher.

Copyright () 2022 Tarrant, Jackson and Viczko. This is an open-access article distributed under the terms of the Creative Commons Attribution License (CC BY). The use, distribution or reproduction in other forums is permitted, provided the original author(s) and the copyright owner(s) are credited and that the original publication in this journal is cited, in accordance with accepted academic practice. No use, distribution or reproduction is permitted which does not comply with these terms. 